\title{
Normalized Analysis for the Sensitivity Optimization of Integrated Optical Evanescent-Wave Sensors
}

\author{
Olivier Parriaux, Associate Member, IEEE, and G. J. Veldhuis
}

\begin{abstract}
Closed-form analytical expressions and normalized charts provide the conditions for the maximum sensitivity of transverse electric (TE) and transverse magnetic (TM) evanescent-wave step-index waveguide sensors. The analysis covers both cases where the measurand is homogeneously distributed in the semi-infinite waveguide cover, and where it is an ultrathin film at the waveguide-cover interface.
\end{abstract}

Index Terms - Biosensors, chemical sensors, optical planar waveguides, refractometers, sensitivity analysis.

\section{INTRODUCTION}

B IOCHEMICAL sensors is the noncommunication application field where integrated optic technology is expected to play an increasing role and where it is already successful commercially [1], [2]. The type of waveguide which is most currently used is a slab structure with a stepindex profile. The sensing is performed by the evanescent tail of the modal field in the cover medium. This sensing operation consists of measuring the change of the effective index of a propagating mode when a change of refractive index takes place in the waveguide cover. The waveguide characteristic equation or/and a calibration allows the retrieval of the index change from the measured change of the effective index. The sensitivity of the measurement of the physical or chemical quantity present in the cover depends on the strength and the distribution of the evanescent field in the cover. The main design task is therefore to find the waveguide structure which maximizes the sensitivity on the quantity to be measured [3]. So far, this has been made in a case-bycase fashion, with each new set of optogeometrical parameters requiring for a remake of the structure modeling. This paper presents a normalized algebraic formalism, allowing for all possible practical cases to be condensed by means of two reduced optogeometrical parameters $a_{s}$ and $a_{c}$ only. The conditions for maximum sensing sensitivity are shown to be contained in a single universal chart where they are represented by a surface $X_{s}\left(a_{s}, a_{c}\right)$ from which all parameters of the optimized structure can be derived. This is the most condensed formalization which the simple electromagnetism of the slab waveguide encompasses.

Manuscript received February 24, 1997; revised December 29, 1997. This work was supported in part by the Twente University project $\mu$ TAS. The work of G. J. Veldhuis was supported by IOP Electro-Optics.

O. Parriaux was with Twente University, MESA Research Institute, NL7500AE Enschede, The Netherlands. He is now with the Laboratoire TSI, UniversitéJean Monnet, F-42023 Saint-Etienne, France.

G. J. Veldhuis is with Twente University, MESA Research Institute, NL7500AE Enschede, The Netherlands.

Publisher Item Identifier S 0733-8724(98)02604-8.

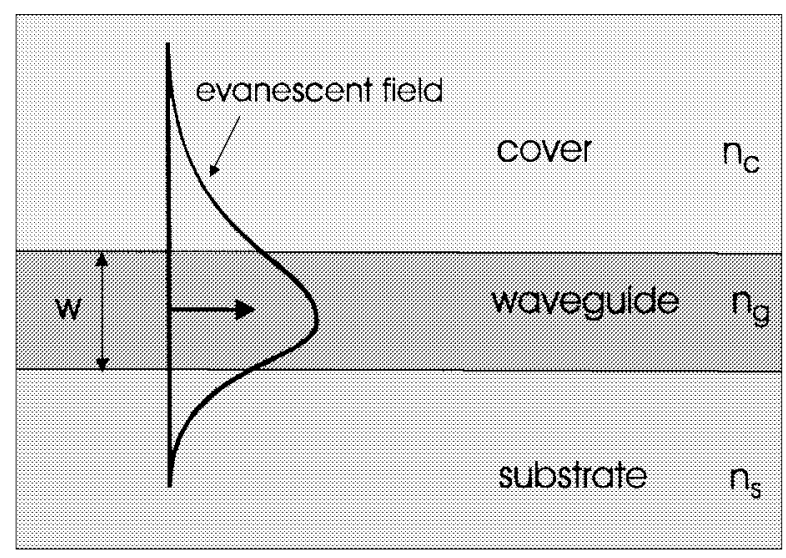

(a)

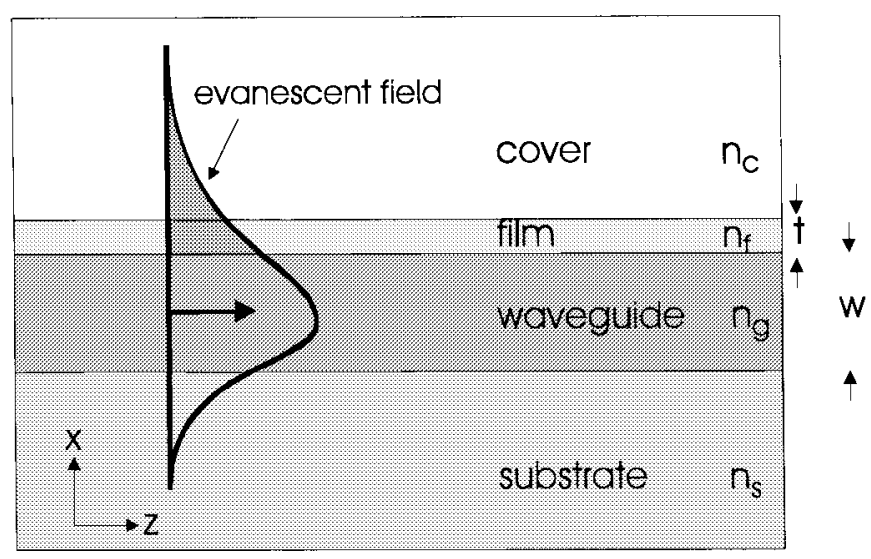

(b)

Fig. 1. Schematic representation of (a) slab waveguide refractometric sensor and (b) surface sensor.

The normalized analysis differs somewhat if the measurand is homogeneously distributed in the cover (afterwards refered to as homogeneous sensing) or it is an ultrathin film at the waveguide-cover interface (surface sensing). Both cases are shown here to be governed by a normalized solution expressed by means of common normalized variables and reduced parameters. They are illustrated schematically in Fig. 1. It is assumed hereafter that the cover medium is a liquid or a gas, which implies that the contact zone between the cover and the waveguide surface is of zero thickness and does not exhibit an air film or bubbles.

So far, planar evanescent-guided wave sensors have mostly been used for the detection of ultrathin biological molecular layers of thickness $t(t \ll \lambda$ where $\lambda$ is the wavelength in vacuum) immobilized on the surface of a waveguide (surface sensing). Such a sensing scheme is currently the subject of 
keen interest in pharmaceutical applications such as imunoassays [3]. It is also of interest in chemical sensing schemes where the opto-chemical transducing mechanism involves an ultrathin surface layer [4]. The sensitivity in such a configuration, illustrated in Fig. 1(b), is related to the squared field magnitude at the waveguide-cover interface [5]. Optimizing the sensor sensitivity requires a suitable choice of the waveguide and substrate index $n_{g}$ and $n_{s}$, respectively, as well as the waveguide thickness $w$ relative to the wavelength $\lambda$, which maximize the squared modal field at the surface. Evanescent wave sensing of a chemical or physical quantity which is homogeneously distributed in the semi-infinite waveguide cover (homogeneous sensing) refers to a different electromagnetic condition. The sensitivity is now related to the integral of the squared evanescent field in the cover material [5]. A waveguide refractometric sensor, as sketched in Fig. 1(a), can be used in liquid concentration monitoring, for measuring traces of chemicals by means of a thick selective membrane [6] and, more generally, for measuring all physical/chemical quantities whose variation corresponds to a change of index. It can also be used for the detection of affinity reactions and immunoassays in cases where the molecules are not immobilized at the very surface of the waveguide, but are homogeneously distributed throughout an organic film whose thickness $t$ is large relative to the penetration depth of the evanescent field.

A normalized expression, giving the condition for maximum transverse electric (TE) sensitivity in the case of both homogeneous and surface sensing, was given in a previous paper [7]. The sensitivity was defined as the rate of change of the modal effective permittivity $\varepsilon_{e}=n_{e}^{2}$ relative to the cover permittivity $\varepsilon_{c}=n_{c}^{2}$. This led to a very simple and homogeneous condition given by a single variable transcendental analytical expression with a single parameter. This parameter was the asymmetry parameter $a=\left(\varepsilon_{g}-\varepsilon_{c}\right) /\left(\varepsilon_{g}-\varepsilon_{s}\right)$ where $\varepsilon_{s}=n_{s}^{2}$ and $\varepsilon_{g}=n_{g}^{2}$ are the square of the substrate and waveguide indexes $n_{s}$ and $n_{g}$, respectively. What really matters in optical detection, such as in an interference [8] or mode synchronism scheme [9], is the mode effective index $n_{e}$ rather than the effective permittivity $\varepsilon_{e}$ because this is the phase of a light wave that is measured, not the dielectric constant. Defining the sensor sensitivity as the rate of change of $n_{e}$ relative to $n_{c}$ (homogeneous sensing) and to the normalized thinfilm thickness (surface sensing) leads to a less normalizable expression. The main aim of the present paper, is to give exact maximum sensitivity expressions for both TE and transverse magnetic (TM) modes in both sensing schemes and to provide a set of universal charts allowing the designer to find the working point of maximum sensitivity and to create the dimensioning of his/her sensor structure.

\section{StRUCTURE ANALYSIS}

\section{A. The Normalized Variables and Reduced Parameters}

The most condensed normalization is made possible by resorting to the normalized effective index $X_{s}$ given by

$$
X_{s}=\sqrt{\frac{\varepsilon_{e}-\varepsilon_{s}}{\varepsilon_{g}-\varepsilon_{e}}} \text { and } X_{c}=\sqrt{\frac{\varepsilon_{e}-\varepsilon_{c}}{\varepsilon_{g}-\varepsilon_{e}}}
$$

which is the associated-dependent variable through

$$
X_{c}^{2}=a\left(1+X_{s}^{2}\right)-1
$$

where

$$
a=\frac{1-a_{c}}{1-a_{s}} \quad \text { and } \quad a_{s}=\frac{\varepsilon_{s}}{\varepsilon_{g}}, \quad a_{c}=\frac{\varepsilon_{c}}{\varepsilon_{g}}
$$

are the two asymmetry parameters. Small values of $a_{s}$ and $a_{c}$ correspond to large guidance structures as in the case of high-index oxide on silica with water cover, and large values of $a_{s}$ and $a_{c}$ correspond to weak guidance structures as in the case of phosphosilicate glass on silica.

Having found the $X_{s}$ value achieving the maximum sensitivity in a given sensing scheme (homogeneous or surface sensing) and for a given polarization, one can retrieve the effective index from (1), and all other optogeometrical quantities of interest by using

$$
n_{e}=n_{g} \sqrt{\frac{a_{s}+X_{s}^{2}}{1+X_{s}^{2}}}
$$

The condition of maximum sensitivity will be simply derived from the waveguide characteristic equation written in terms of the normalized, mutually dependent variables $X_{s}$ and $X_{c}$ :

In the homogeneous sensing case, represented in Fig. 1(a)

$$
k_{0} w n_{g} \sqrt{\frac{1-a_{s}}{1+X_{s}^{2}}}-\arctan \xi_{s} X_{s}-\arctan \xi_{c} X_{c}-m \pi=0
$$

with $\xi_{s}=\xi_{c}=1$ for TE and $\xi_{s}=a_{s}^{-1}, \xi_{c}=a_{c}^{-1}$ for TM modes. $m$ is the mode order, $k_{0}=2 \pi / \lambda$ is the free space wave number and $w$ is the slab waveguide thickness.

In the surface sensing case, represented in Fig. 1(b), the writing of a condensed characteristic equation requires some preparation. The structure consists of four homogeneous media in which the $E_{x}$ field for TE modes, and the $H_{x}$ for TM modes, are expressed by a superposition of two exponential functions. In the waveguiding layer, the argument of the exponentials is imaginary. In the substrate and in the cover it is real and the function is selected which corresponds to a confined waveguide field. In the ultrathin film of thickness $t$, the field is, in turn, represented as a superposition of two exponential functions of coordinate $y$. These have an imaginary or real argument depending on whether the mode effective index $n_{e}$ is smaller or larger than the film index $n_{f}$. In most cases of current interest, the ultrathin film consists of biological molecules having an index of around 1.4 on top of a waveguide of much larger refractive index. In the algebraic derivations which follow, we shall limit ourselves to an optical thickness of the film $k_{0} t \sqrt{\varepsilon_{e}-\varepsilon_{f}}$ much smaller than 1. Consequently

$$
\exp \left( \pm k_{0} t \sqrt{\varepsilon_{e}-\varepsilon_{f}}\right) \cong 1 \pm k_{0} t \sqrt{\varepsilon_{e}-\varepsilon_{f}}
$$

The matching of the four tangential field components at all three interfaces yields, for the TE and TM polarizations, six linear equations involving the six unknown integration constants. The condition for a nondegenerate solution leads to a characteristic equation which, using (6), takes the familiar 
form of the three-layer equation with, however, a perturbation term $\delta$ accounting for the presence of the ultrathin film

$$
\begin{aligned}
& k_{0} w n_{g} \sqrt{\frac{1-a_{s}}{1+X_{s}^{2}}}-\arctan \xi_{s} X_{s} \\
& -\arctan \xi_{c}(1-\delta) X_{c}-m \pi=0
\end{aligned}
$$

where

$$
\delta=k_{0} t\left(\varepsilon_{f}-\varepsilon_{c}\right) \quad \text { for TE modes }
$$

and

$$
\delta=k_{0} t\left(\varepsilon_{f}-\varepsilon_{c}\right) \frac{\varepsilon_{e}\left(1 / \varepsilon_{c}+1 / \varepsilon_{f}\right)-1}{\sqrt{\varepsilon_{e}-\varepsilon_{c}}} \text { for TM modes. }
$$

The derivation shows that (7) and (8) hold in both cases for $\varepsilon_{f}<\varepsilon_{e}$ and $\varepsilon_{f}>\varepsilon_{e}$.

\section{Sensitivity Evaluation}

In the case of homogeneous sensing, the sensitivity $S_{h}$ is defined as the rate of change of the modal effective index $n_{e}$ under an index change of the cover. The sensitivity $S=$ $\left(\partial n_{e} / \partial n_{c}\right)$ of a structure of constant $w k_{0}, n_{g}, n_{c}$, and $n_{s}$ is first calculated explicitly $\left(\partial n_{c} / \partial n_{e}\right)^{-1}$ as for both TE and TM modes

$$
\begin{aligned}
S_{h, \mathrm{TE}}= & \sqrt{a_{c}}\left\{X_{c} \sqrt{1+X_{c}^{2}} \sqrt{a_{c}+X_{c}^{2}}\right. \\
& \left.+\left[\frac{1}{X_{c}}+\frac{1}{X_{s}} \arctan X_{s}+\arctan X_{c}+m \pi\right]\right\}^{-1}
\end{aligned}
$$$$
S_{h, \mathrm{TM}}=\frac{\frac{2}{\sqrt{a_{c} q}}-\sqrt{a_{c} q}}{1+X_{c}^{2}+r \cdot F}
$$

where

$$
q=\frac{1+X_{s}^{2}}{a_{s}+X_{s}^{2}}, \quad r=\frac{X_{c}}{a_{c}}\left(a_{c}^{2}+X_{c}^{2}\right)
$$

and

$$
F=\arctan \frac{X_{s}}{a_{s}}+\arctan \frac{X_{c}}{a_{c}}+m \pi+\frac{a_{s}\left(1+X_{s}^{2}\right)}{X_{s}\left(a_{s}^{2}+X_{s}^{2}\right)} .
$$

Expressions (9) and (10) give the analytical form of the sensitivity of a mode of order $m$ versus the parameters $n_{g}, n_{s}$, and $n_{c}$. The parameter $w k_{0}$ does not appear explicitly, because the characteristic equations in (5) were used to express $w k_{0} n_{g}$ as satisfying the electromagnetic boundary conditions.

The sensitivity $S_{S}$ of a surface sensing structure is defined as the rate of change of the modal effective index $n_{e}$ versus the dielectric load term that is defined as

$$
\eta=k_{0} t\left(\varepsilon_{f}-\varepsilon_{c}\right) .
$$

No explicit direct relationship $S_{s}=\partial n_{e} / \partial \eta$ can be obtained. Therefore, $S_{s}=\left(\partial \eta / \partial n_{e}\right)^{-1}$ was calculated from (7) analytically. This results are in (12) shown at the bottom of the page and

$$
S_{s, \mathrm{TM}}=\frac{\left(p \frac{X_{c}^{2}+a_{c}}{1+X_{c}^{2}}-1\right) \frac{a_{c}}{a_{c}^{2}+X_{c}^{2}} \sqrt{\frac{1-a_{c}}{a_{c}+X_{c}^{2}}}}{F}
$$

where

$$
\begin{gathered}
F=\arctan \frac{X_{s}}{a_{s}}+\arctan \frac{X_{c}}{a_{c}}+m \pi \\
+\frac{a_{s}\left(1+X_{s}^{2}\right)}{X_{s}\left(a_{s}^{2}+X_{s}^{2}\right)}+\frac{a_{c}\left(1+X_{c}^{2}\right)}{X_{c}\left(a_{c}^{2}+X_{c}^{2}\right)} . \\
p=\frac{1}{a_{f}}+\frac{1}{a_{c}}, \quad a_{f}=\frac{\varepsilon_{f}}{\varepsilon_{g}} .
\end{gathered}
$$

An important distinction must be made between TE and TM modes. The normalized sensitivity of TE modes on the dielectric load $\eta$ is independent of $\varepsilon_{f}$. This means that the expressions (12) of $S_{s, \mathrm{TE}}$ is generally valid regardless of the type and weight of dielectric load as long and $\eta \ll 1$. This is not exactly true for the TM modes since $S_{s, \mathrm{TM}}$ (expression (13)) contains the thin film permittivity $\varepsilon_{f}$ in $p$. This infers that a normalization of the TM problem by means of two parameters only is not completely possible. Nevertheless, it will be shown later that the condition for maximum sensitivity can be given with a satisfactory accuracy by a general twoparameter solution.

\section{The Condition for MaXimum Sensing Sensitivity}

Searching for the condition of maximum sensitivity in a structure of constant $n_{g}, n_{s}$, and $n_{c}$ amounts to canceling the derivative of $S_{\mathrm{TE}}$ and $S_{\mathrm{TM}}$ with respect to $k_{0} w$. After some algebraic manipulations (using $\delta S / \delta\left(k_{0} w\right)=\delta S / \delta X_{s}$. $\delta X_{s} / \delta k_{0} w$ and $\left.\delta X_{s} / \delta\left(k_{0} w\right) \neq 0\right)$ the exact maximum condition can be obtained.

In the homogeneous sensing case

$$
\begin{aligned}
& {\left[\frac{1}{X_{c}}+\frac{1}{X_{s}}+\arctan X_{c}+\arctan X_{s}+m \pi\right]} \\
& \cdot\left[\frac{1}{X_{c}^{2}}+3+\frac{\left(1-a_{s}\right)}{\left(a_{s}+X_{s}^{2}\right)}\right]-\frac{1}{X_{c}^{3}}-\frac{1}{X_{s}^{3}}=0
\end{aligned}
$$

for TE modes, while for TM modes, we find (15) shown at the bottom of the next page where

$$
t=\frac{1-a_{c}}{1-a_{s}} \frac{X_{s}}{X_{c}}
$$

Since $X_{c}=X_{c}\left(X_{s}, a_{c}, a_{s}\right)$, expressions (14) and (15) are single variable $\left(X_{s}\right)$, transcendental equations with two parameters, $a_{s}$ and $a_{\mathcal{c}}$, which can be solved numerically once and for all.

$$
S_{s, \mathrm{TE}}=\frac{\sqrt{1-a_{s}}}{\sqrt{a_{s}+X_{s}^{2}}\left(1+X_{c}^{2}\right)\left[\arctan X_{s}+\arctan X_{c}+m \pi+\frac{1}{X_{c}}+\frac{1}{X_{s}}\right]}
$$


In the case of surface sensing, we have the following. For TE modes

$$
\begin{aligned}
& {\left[\arctan X_{c}+\arctan X_{s}+m \pi+\frac{1}{X_{c}}+\frac{1}{X_{s}}\right]} \\
& \cdot\left(3+\frac{1-a_{s}}{a_{s}+X_{s}^{2}}\right)-\frac{1}{X_{c}^{3}}-\frac{1}{X_{s}^{3}}=0 .
\end{aligned}
$$

As expected, expression (16) is very close to the expression given in [7] where the present term $\left(3+1-a_{s}\right) /\left(a_{s}+X_{s}^{2}\right)$ was simply given by a factor of three which is sufficiently accurate for structures having $a_{s}$ close to one, i.e., in weakly guiding waveguides.

Searching for the condition of maximum sensitivity for TM modes is more cumbersome. Nevertheless, a normalized expression can be given

$$
\begin{aligned}
& \left(p \frac{a_{s}+X_{s}^{2}}{1+X_{s}^{2}}-1\right) \\
& \cdot\left\{\frac{1-a_{c}}{1-a_{s}} X_{s}\left[\frac{1}{X_{c}}\left(2-\frac{1}{X_{c}^{2}}\right)+\frac{2 G}{a_{c}}\right]\right. \\
& \left.+\frac{a_{s}\left(a_{c}^{2}+X_{c}^{2}\right)}{a_{c} X_{s}^{2}\left(a_{s}^{2}+X_{s}^{2}\right)^{2}}\left(2 a_{s}^{2} X_{s}^{2}-3 X_{s}^{2}-a_{s}^{2}\right)\right\} \\
& -X_{s}\left[\frac{1}{a_{s}+X_{s}^{2}}+\frac{p}{\left(1+X_{s}^{2}\right)^{2}}\left(1-2 a_{s}-X_{s}^{2}\right)\right] \\
& \quad \times \frac{a_{c}^{2}+X_{c}^{2}}{a_{c}} F=0
\end{aligned}
$$

where

$$
G=F-\frac{a_{c}}{X_{c}} \frac{1+X_{c}^{2}}{a_{c}^{2}+X_{c}^{2}} .
$$

Again, as in the TM sensitivity expression, the film-dependent term $n_{f}$ is present in expression (17) as contained in the $p$ term.

At this stage a remark on symmetry should be made which extends the scope of the above expressions. If the ultrathin film is placed at the guide-substrate interface, the sensitivity expressions (12), (13), as well as (16), and (17) have the same structure where $a_{s}, a_{c}, X_{s}$, and $X_{c}$ are substituted by $a_{c}, a_{s}$, $X_{c}$, and $X_{s}$, respectively. In the expressions for $\eta(11), p$, and $G, \varepsilon_{c}$ is replaced by $\varepsilon_{s}$.

In both homogeneous and surface sensing cases the normalized waveguide thickness achieving maximum sensitivity can be obtained by substituting the $X_{s}$ solutions of (14)-(17) into the characteristic equation of the three-layer structure for $\mathrm{TE}_{0}$ and $\mathrm{TM}_{0}$ modes

$$
\frac{n_{g} w}{\lambda}=\frac{1}{2 \pi} \sqrt{\frac{1+X_{s}^{2}}{1-a_{s}}}\left[\arctan \xi_{s} X_{s}+\arctan \xi_{c} X_{c}\right] .
$$

\section{REPRESENTATION OF THE CONDITIONS For MaXimum Sensing Sensitivity}

Equations (14)-(17) alone contain the condition for maximum sensitivity of every possible step index evanescent wave sensor of the homogeneous and surface sensing type using $\mathrm{TE}$ and TM modes. The procedure to retrieve the waveguide parameters ensuring maximum sensitivity, and to find the maximum that can be achieved, is as follows.

The solution to each of the transcedental equations (14)-(17) is one value of the normalized refractive index $X_{s}$ for each pair of asymmetry parameters $\left(a_{s}, a_{c}\right)$. The effective index $n_{e}$ to which an $X_{s}$ solution corresponds, is given by expression (4). The normalized thickness $n_{g} w / \lambda$ of the waveguide ensuring maximum sensitivity is found by substituting the $X_{s}$ solution into expression (18). It is interesting at that stage to locate the optimum value of $n_{g} w / \lambda$ with respect to the cut-off of the sensing mode (usually the fundamental mode) and to the cut-off of the higher-order modes. These cut-off values can also be expressed in terms of the normalized parameters from expression (5) with $X_{s}=0$

$$
\left(\frac{n_{g} w}{\lambda}\right)_{\text {cut-off }}=\frac{1}{2 \pi \sqrt{1-a_{s}}} \arctan \xi_{c} \sqrt{\frac{a_{s}-a_{c}}{1-a_{s}}} .
$$

The maximum achievable sensitivity is obtained by substituting the $X_{s}$ solution into the sensitivity expressions (9) and (10) in the homogeneous sensing case and (12) and (13) in the surface sensing case. The general solution obtained here can be easily and advantageously represented graphically in 3-D charts where the optimized quantities of interest $X_{s}$, $n_{g} w / \lambda$ and $S$ define a surface, all three being functions of two variables only, namely, $a_{s}$ and $a_{c}$. This vivid graphical representation is also a complete one since it contains all information on the optimized structures whatever the set of refractive indexes, the wavelength or the waveguide width may be. The discussion which follows will be based on the features revealed by the charts.

All together there are two sensing schemes (homogeneous and surface sensing) and two polarizations (TE and TM modes). This is assuming that the sensing is performed by the fundamental mode, which is most often the case because it has the highest sensitivity. However, our analytical expressions are valid for any mode order $m$. Each of the four cases is fully described by a set of three charts $X_{s}\left(a_{s}, a_{c}\right), n_{g} w / \lambda\left(a_{s}, a_{c}\right)$,

$$
\begin{gathered}
\left(1+X_{c}^{2}+r \cdot F\right)\left(2 X_{s} \frac{1-a_{s}}{\left(a_{s}+X_{s}^{2}\right)^{2}} \frac{1}{\sqrt{a_{c} q}}\left(\frac{1}{q}+\frac{a_{c}}{2}\right)\right)-\left(\frac{2}{\sqrt{a_{c} q}}-\sqrt{a_{c} q}\right) \\
\cdot\left(2 X_{c} \cdot t+F \cdot \frac{1}{a_{c}}\left(a_{c}^{2}+3 X_{c}^{2}\right) \cdot t+r \cdot\left(\frac{a_{s}}{a_{s}^{2}+X_{s}^{2}}+\frac{a_{c}}{a_{c}^{2}+X_{c}^{2}} \cdot t a_{s}\left(\frac{2 X_{s}^{2}\left(a_{s}^{2}+X_{s}^{2}\right)-\left(1+X_{s}^{2}\right)\left(a_{s}^{2}+3 X_{s}^{2}\right)}{X_{s}^{2}\left(a_{s}^{2}+X_{s}^{2}\right)^{2}}\right)\right)\right)=0
\end{gathered}
$$




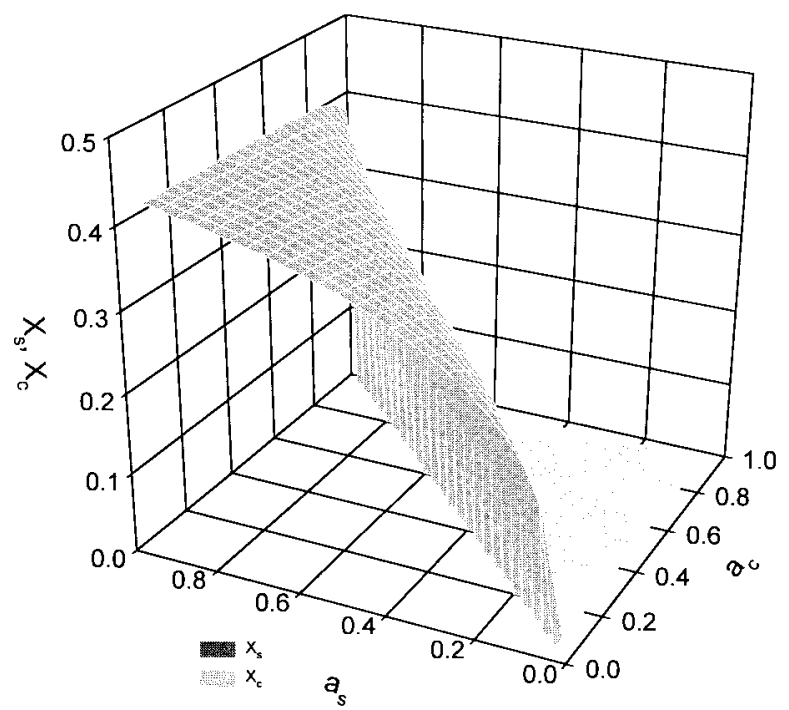

Fig. 2. Normalized effective refractive index solution $X_{s}$ versus $a_{s}$ and $a_{c}$ ensuring maximum sensitivity for refractometric sensing using the $\mathrm{TE}_{0}$ mode. $X_{c}=0$ is represented in the plane sector $a_{c}>a_{s}$.

and $S\left(a_{s}, a_{c}\right)$. From now on, the discussion of the sensing features will be made on a case-by-case basis.

The reader who wishes to use the normalized results obtained above can either plot the $X_{s}\left(a_{s}, a_{c}\right)$-, $n_{g} w / \lambda\left(a_{s}, a_{c}\right)$ and $S\left(a_{s}, a_{c}\right)$-charts from the previous equations, or just look at the plots of Figs. 2-14 which contain all the necessary information on his/her optimized sensor structure. A typical way of proceeding is as follows.

Usually the sensor usage imposes the cover material, thus its nominal index $n_{c}$. The substrate material results from a choice involving temperature and mechanical stability, and also cost criteria, which imposes $n_{s}$. There is usually a relative freedom in the choice of the waveguide material, the main demands on it being the optical and chemical stability. Thus, $n_{g}$ remains a free parameter, as well as the ratio $w / \lambda$ whereas $a_{s}$ and $a_{c}$ are fixed. The designer will first look at the maximum sensitivity chart corresponding to his/her type of sensor (homogeneous or surface sensing) and to the possibly preferred polarization. In this $S$-chart, the designer will search for the $\left(a_{s}, a_{c}\right)$ coordinates (remember $a_{s}=n_{s}^{2} / n_{g}^{2}$ $a_{c}=n_{c}^{2} / n_{g}^{2}$ ) which provides the highest sensitivity among the various waveguide refractive indexes that are available. Having determined the optimum $\left(a_{s}, a_{c}\right)$ coordinate, he/she will go to the corresponding $n_{g} w / \lambda$-chart where the just found $\left(a_{s}, a_{c}\right)$ coordinate will determine the $n_{g} w / \lambda$-value ensuring maximum sensitivity. Having already determined $n_{g}$, he/she can now retrieve the relative waveguide thickness $w / \lambda$ which the waveguide must have to set the sensor at its best working point.

\section{Discussion ON THE MAXIMUM SENSING CONDITIONS}

\section{A. Homogeneous Sensing}

The sensing configuration is represented in Fig. 1(a). The TE and TM modes will be discussed separately.

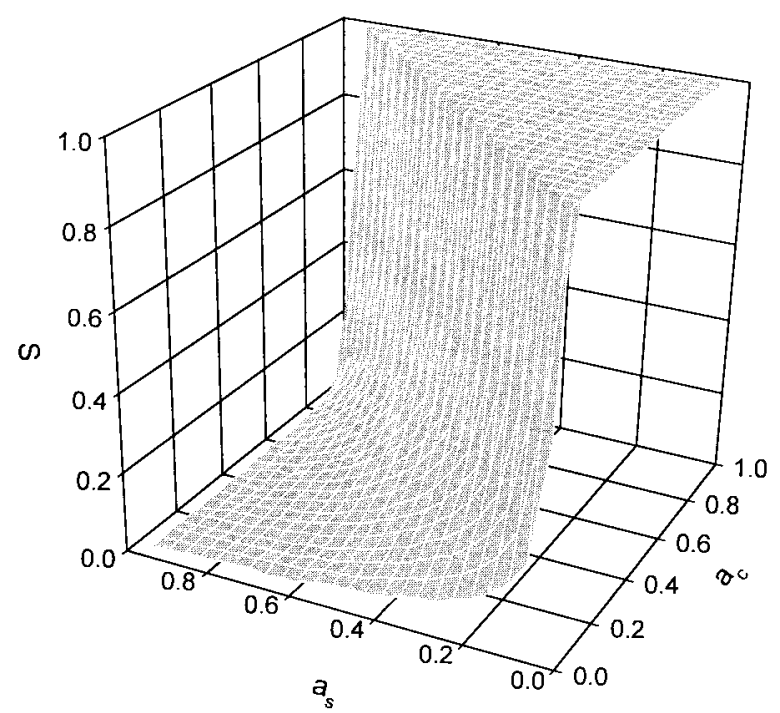

Fig. 3. Maximum achievable sensitivity for $\mathrm{TE}_{0}$ refractometric sensing versus $a_{s}$ and $a_{c} . S=1$ for $a_{c}>a_{s}$.

1) TE-Polarization: Fig. 2 is the chart of the condition on $X_{s}$ for maximum sensitivity of the $\mathrm{TE}_{0}$ mode [expression (14)]. Introducing the solution found for $X_{s}$ into expression (9) gives the maximum sensitivity $S_{\mathrm{TE}}$ achievable in a waveguide structure characterized by the asymmetry parameters, $a_{s}$ and $a_{c} . S_{\mathrm{TE}}$ is similarly represented as a surface in the $S_{\mathrm{TE}}\left(a_{s}, a_{c}\right)$ chart of Fig. 3. Whereas Fig. 2 shows the condition for maximum sensitivity and Fig. 3 illustrates the maximum achievable sensitivity, the last chart (Fig. 4) tells the designer the corresponding normalized optical thickness $n_{g} w / \lambda$ [expression (18) with $\xi_{s}=\xi_{c}=1$ ]. The designer is interested in evaluating how far his/her optimum sensitivity configuration is from the cutoff condition of the zeroth- and first-order modes given by $m=0$ and 1 in the cutoff expression (19) with $\xi_{c}=1$. These two cutoff surfaces are also shown on the chart in the form of a light mesh.

The surface of Fig. 2 comprises two sections. The section where $a_{s}>a_{c}$ corresponds to the case most often met in practice of a substrate index larger than the analyte index, often a water based solution or a polymeric material. $X_{s}\left(a_{s}, a_{c}\right)$ is only slightly dependent on $a_{c}$ for large $a_{s}$ values. In all cases $X_{s}$ tends to zero as $a_{s}$ tends to $a_{c}$. The limit is the cut-off situation in a symmetrical waveguide, which corresponds to $n_{g} w / \lambda=0$ in Fig. 4. In this case, the modal field extends symmetrically into the substrate and the cover, which implies that the maximum sensitivity is 0.5 as clearly indicated in the sensitivity chart of Fig. 3. The section of all three charts of Figs. 2-4 where $a_{s}<a_{c}$, corresponds to the case of a cover index larger than that of the substrate. Here, the condition of maximum sensitivity on the cover's index is the cutoff condition, where $X_{c}=0$ ( $X_{c}$ is represented in Fig. 2 instead of $X_{s}$ when $a_{s}<a_{c}$, which takes values that are too large to be properly presented), $S_{\mathrm{TE}}=1$ in Fig. 3 , and $n_{g} w / \lambda=n_{g} w / \lambda_{\text {cutoff }}$ in Fig. 4 .

As mentioned earlier, the index configuration most often met in practice is that of $a_{s}>a_{c}$, in which case a definite maximum sensitivity exists. An interesting feature of the 


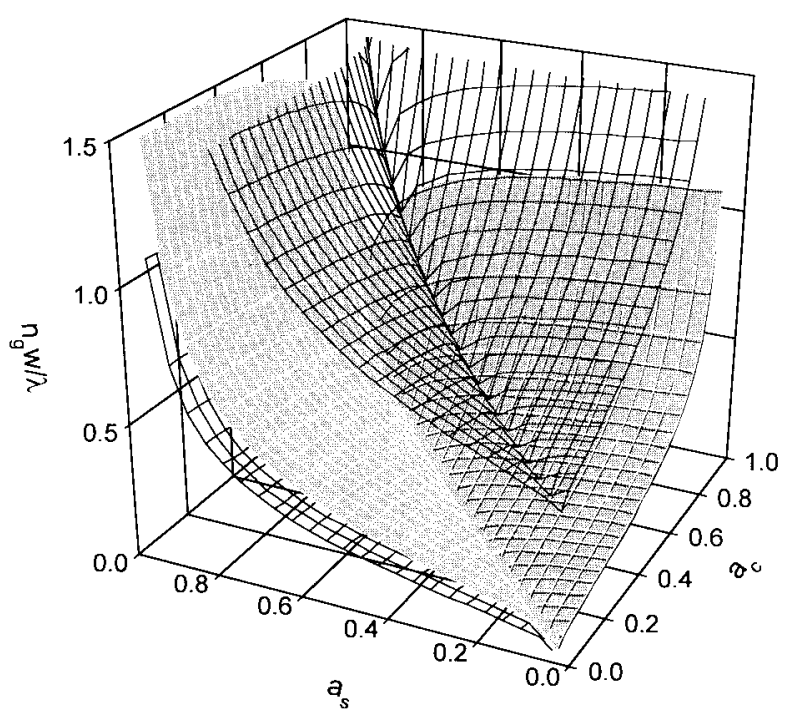

Fig. 4. Optical thickness $n_{g} w / \lambda$ ensuring the maximum sensitivity for $\mathrm{TE}_{0}$ refractometric sensing versus $a_{s}$ and $a_{c}$. The cutoff condition for the $\mathrm{TE}_{0}$ and the $\mathrm{TE}_{1}$ modes are indicated by the mesh. The surface for $a_{c}>a_{s}$ is the $\mathrm{TE}_{0}$ cutoff surface.

sensitivity chart is that, contrary to what is often believed as a false analogy with surface sensing, achieving maximum sensitivity does not require the use of a large guidance waveguide technology. A comparable sensitivity can be obtained by weak guidance waveguides which generally present lower scattering losses and better chemical stability.

The chart in Fig. 4 shows that the condition of maximum sensitivity for $n_{g} w / \lambda$ is quite close to the fundamental mode cutoff. This can be estimated by comparing the surface of the section where $a_{s}>a_{c}$ with the cutoff surface of the other section $\left(a_{s}<a_{c}\right)$. The configuration for maximum sensitivity tends continuously to the cutoff condition identity when $a_{s} / a_{c}$ approaches one; this is also the region of the highest achievable sensitivity.

2) The TM Polarization: Figs. 5 and 6 convey the same information on the $\mathrm{TM}_{0}$ mode as Figs. 3 and 4 on the $\mathrm{TE}_{0}$ mode. For the sake of conciseness we will skip the $X_{s}\left(a_{s}, a_{c}\right)$ charts giving the normalized effective index ensuring maximum sensitivity, i.e., the solutions of (15) and (17). The maximum sensitivity also occurs in a symmetrical configuration $\left(a_{s}=\right.$ $\left.a_{c}\right)$. As can be seen by comparing the sections where $a_{s}>$ $a_{c}$ of Fig. $5\left(S_{\mathrm{TM}}\left(a_{s}, a_{c}\right)\right.$ maximum achievable sensitivity) with Fig. $6\left(\frac{n_{g} w}{\lambda}\left(a_{s}, a_{c}\right)\right.$ ensuring maximum sensitivity), the $\left(a_{s}, a_{c}\right)$ region of high-maximum sensitivity in the TM case corresponds to a waveguide configuration far from cut-off. This is the reverse of what takes place in the case of the $\mathrm{TE}_{0}$ mode. This is particularly interesting when considering waveguide excitation by means of a grating at the cover side, as well as regarding losses due to substrate waviness.

There is another interesting feature in the relative behavior of the $\mathrm{TE}_{0}$ and $\mathrm{TM}_{0}$ modes. By comparing the values for $S$ found for both polarizations it can be concluded that in the area which is the most interesting in practice, e.g., where $a_{c}<a_{s}$, the $\mathrm{TM}_{0}$ mode is always more sensitive than the $\mathrm{TE}_{0}$ mode. Considering that the $\mathrm{TM}_{0}$ mode's maximum sensitivity occurs for a larger waveguide thickness, away from cutoff, leads us

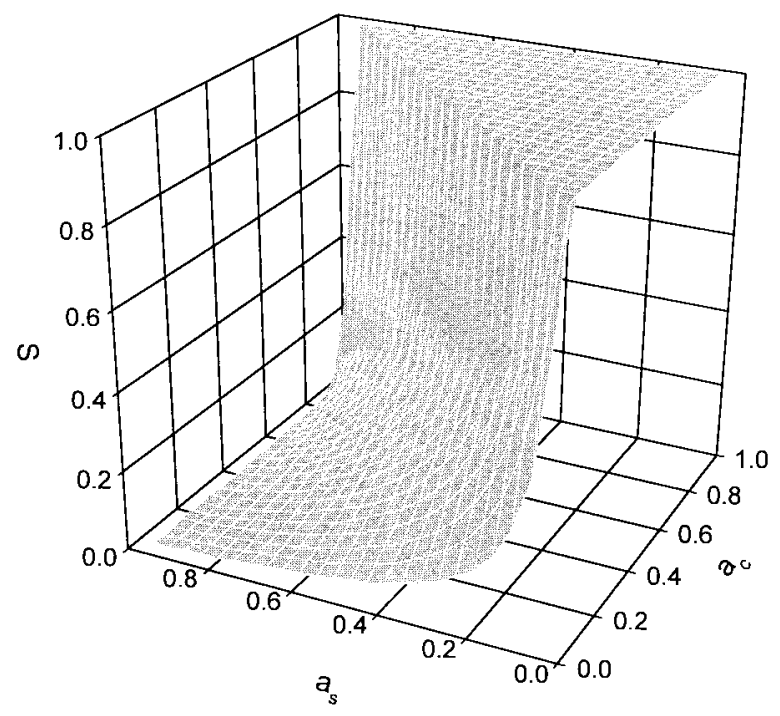

Fig. 5. Maximum achievable sensitivity for $\mathrm{TM}_{0}$ refractometric sensing versus $a_{s}$ and $a_{c} . S=1$ for $a_{c}>a_{s}$.

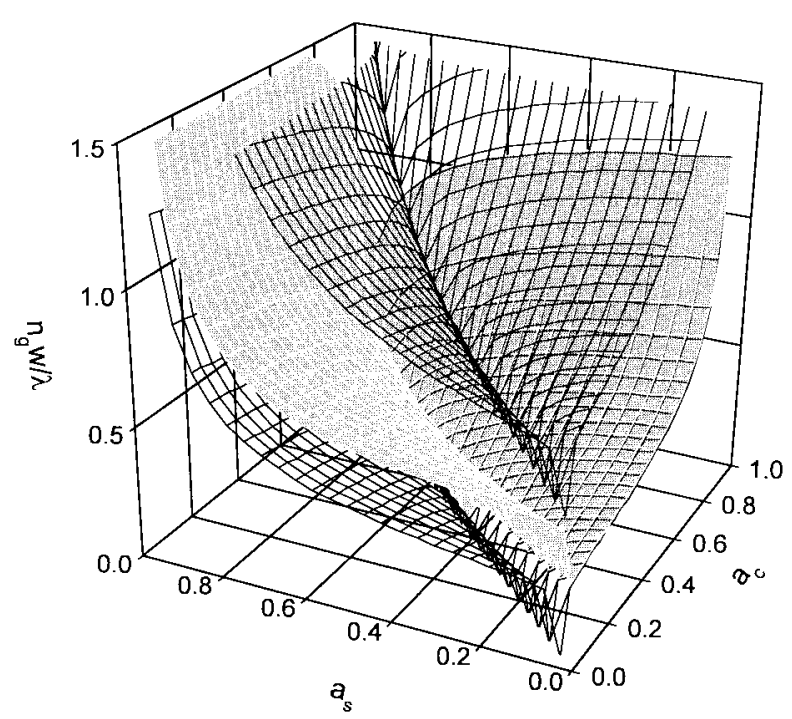

Fig. 6. Optical thickness $n_{g} w / \lambda$ ensuring the maximum sensitivity for $\mathrm{TM}_{0}$ refractometric sensing versus $a_{s}$ and $a_{c}$. The cutoff condition for the $\mathrm{TM}_{0}$ and the $\mathrm{TM}_{1}$ modes are indicated by the mesh. The surface for $a_{c}>a_{s}$ is the $\mathrm{TM}_{0}$ cutoff surface.

to the conclusion that for most practical sensing cases it is advantageous to use the $\mathrm{TM}_{0}$ mode.

3) Generalization: The formalism and expressions derived here in the homogeneous sensing scheme are more general and are not limited to lossless structures. No restrictive hypothesis was made on the permittivity of the different layers, which may be complex. This in particular concerns the absorptive variant of the evanescent wave sensor considered above. If a variation of the measurand translates into an imaginary part of the cover's index change $\Delta n_{c}$, the resulting effective index change $\Delta n_{e}$ generally remains given by the relationship

$$
\Delta n_{e}-S \cdot \Delta n_{c}
$$

Under the hypothesis that the nominal losses of an absorptive sensor are small (i.e. the imaginary part of the cover's 


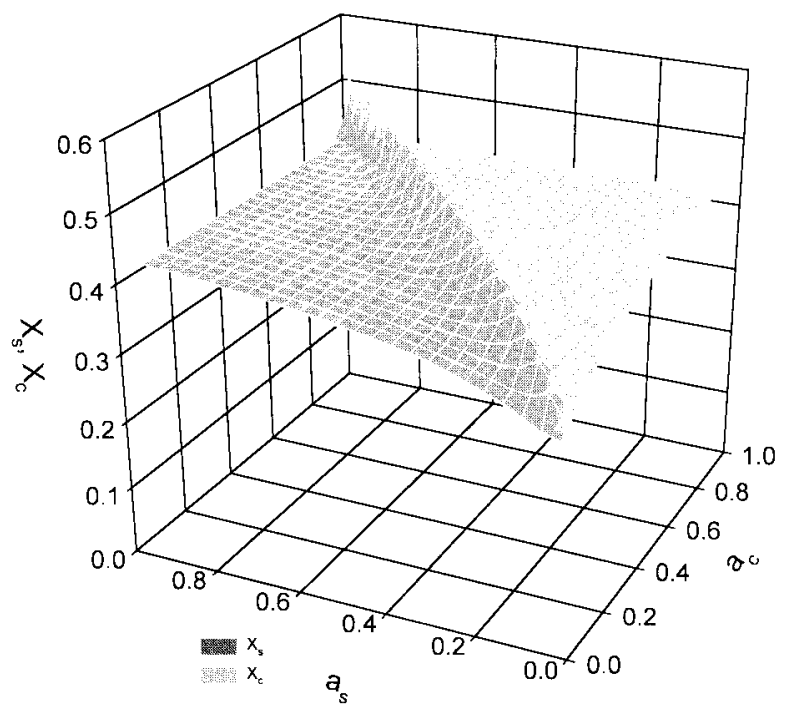

Fig. 7. Normalized effective index solution versus $a_{s}$ and $a_{c}$ ensuring maximum sensitivity for surface sensing using the $\mathrm{TE}_{0}$ mode. For ordinate scale reason, $X_{s}$ is represented in the area $a_{s}>a_{c}$ while $X_{c}$ is represented in the area $a_{c}>a_{s}$.

index is much smaller than the real part, which is true in most cases of interest) the condition for maximum sensitivity for both polarizations is still given by the solutions to (14) and (15) with the real part of the cover's permittivity.

Another generalization of the results obtained in the homogeneous sensing case can be made by considering that the substrate can be a metal. This will simply translate in $a_{s}$ being a complex parameter with a large negative real part. This case is not included in the charts but can be obtained similarly by searching for the zeros of expressions (14) and (15) with zero imaginary part, which will suffice as long as not too lossy metals are used. Such a structure presents a practical interest in sensors where both plasmon and dielectric waveguide modes are used.

\section{B. Surface Sensing}

The following case refers to Fig. 1(b).

1) TE Polarization: Fig. 7 is the chart of the $X_{s}$ solutions of expression (16) ensuring maximum sensitivity of the effective index on the dielectric load $\eta$ for the $\mathrm{TE}_{0}$ mode. The corresponding surface comprises two sections. The first section represents $X_{s}$ in all cases where $a_{s}>a_{c}$. A typical case belonging to this section is that of a silica-based waveguide with a water cover, the thin film being at the guide-cover interface. The second section corresponds to examples where $a_{c}>a_{s}$, the film being still at the cover's interface. In order to make the chart clearer in this section, $X_{c}$ is represented instead of $X_{s}\left(X_{c}=\sqrt{\left.\left(1-a_{c} / 1-a_{s}\right)\left(1+X_{s}^{2}\right)-1\right)}\right.$; the values for $X_{s}$ corresponding to this case would indeed be too large to be illustrated graphically. It is worth pointing out that a definite maximum sensitivity exists whatever the combination of $a_{c}$ and $a_{s}$ unlike in the homogeneous sensing structure (Fig. 2). It can be seen that the larger the guidance and the closer $a_{c}$ to $a_{s}$, the larger the $X_{s}$ value is, i.e., the $n_{e}$ value ensuring sensitivity maximum. The $X_{s}$ values for the $\mathrm{TM}_{0}$ mode are

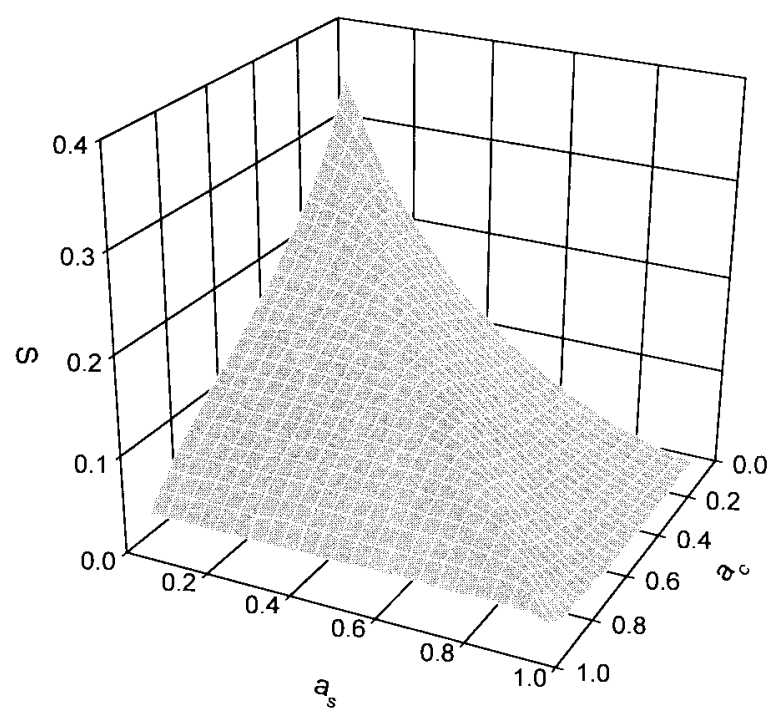

Fig. 8. Maximum achievable sensitivity for $\mathrm{TE}_{0}$ surface sensing versus $a_{s}$ and $a_{c}$.

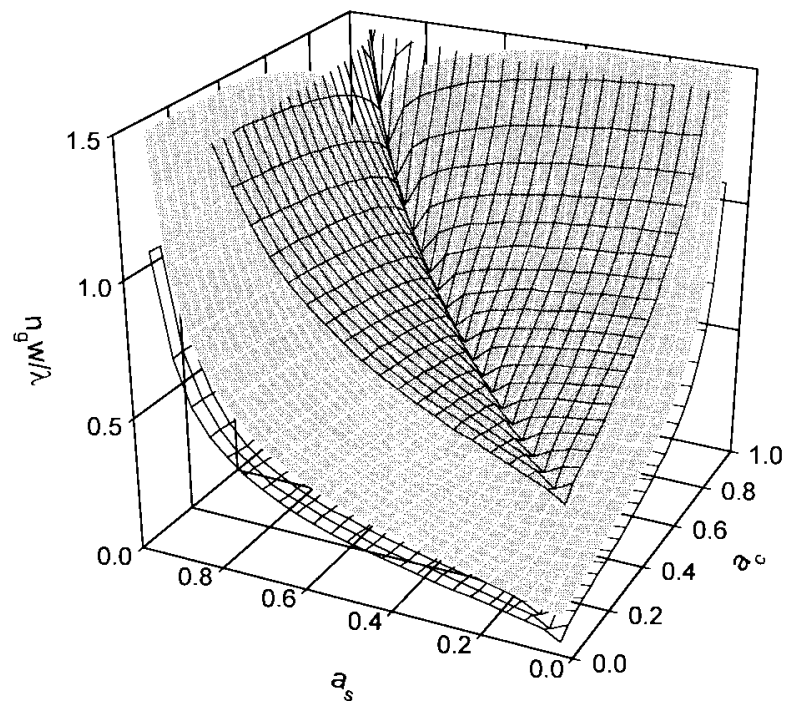

Fig. 9. Optical thickness $n_{g} w / \lambda$ ensuring the maximum sensitivity for $\mathrm{TE}_{0}$ surface sensing versus $a_{s}$ and $a_{c}$. The cutoff condition for the $\mathrm{TE}_{0}$ and the $\mathrm{TE}_{1}$ modes are indicated by the mesh.

generally larger than that of the $\mathrm{TE}_{0}$ mode. Introducing the $X_{s}$ solutions of (16) into the sensitivity expression $S_{\mathrm{TE}}$ (12) leads to the maximum sensitivity chart of Fig. 8, which shows that the maximum sensitivity, increases monotonically with the strength of the guidance at both waveguide sides and that the maximum is obtained for $n_{c}=n_{s}$ (symmetrical waveguide) as in the case of homogeneous sensing. The surface is not symmetrical, showing that the maximum sensitivity is slightly larger when the thin film is at the side of the larger cladding index, obeying $S_{\mathrm{TE}}\left(a_{c}, a_{s}\right)=\left(1-a_{c}\right) /\left(1-a_{s}\right) \cdot S_{\mathrm{TE}}\left(a_{s}, a_{c}\right)$.

Fig. 9 is the chart showing the normalized optical thickness $n_{g} w / \lambda$ of the waveguide for maximum $\mathrm{TE}_{0}$ sensitivity versus $a_{s}$ and $a_{c}$. It is obtained by substituting the $X_{s}$ solutions of (16) into (18). In contrast with the homogeneous sensing case (Fig. 4), maximum sensitivity is reached at a definite distance from the cut-off thickness. 


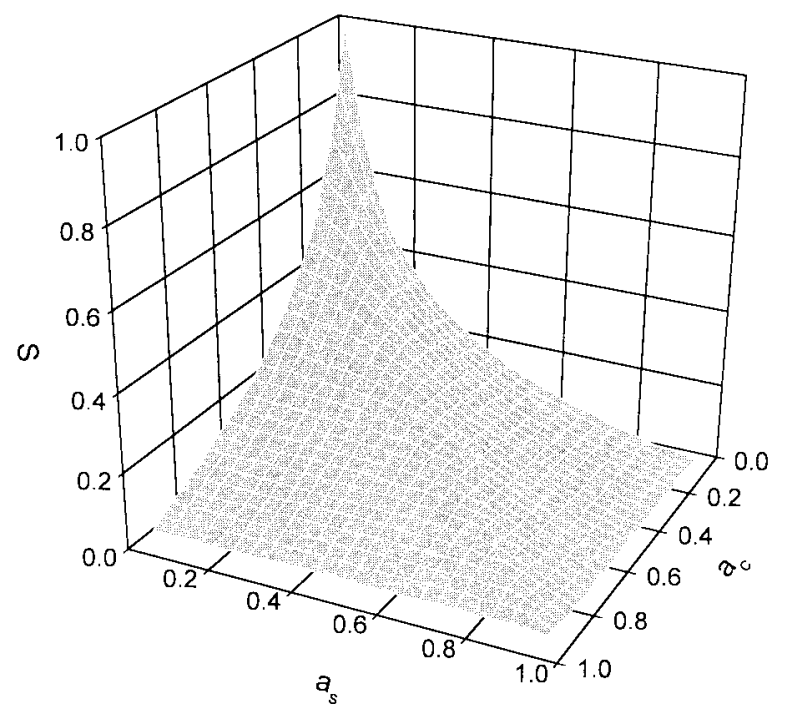

Fig. 10. Maximum achievable sensitivity for $\mathrm{TM}_{0}$ surface sensing versus $a_{s}$ and $a_{c}$, corresponding to $p=2 / a_{c}$.

2) TM Polarization: As pointed out above, the TM polarization analysis is not as straightforward as that of the TE polarization because the sensitivity expression (13) and the condition for maximum sensitivity (17) depend on the dielectric constant $\varepsilon_{f}$ of the measurand. We will, nevertheless, try to obtain conclusions which are as general as possible. To start with, consider the simple electromagnetic case where the index of the film is equal to that of the cover, which case corresponds to $p=2 / a_{c}$. It is not too far from the reality of biosensing since, the index of biospecies, $n_{f}$, is often taken as 1.40-1.45 which is close to the solvent index, $n_{c}=1.33$. Figs. 10 and 11, which correspond to Figs. 8 and 9 for $\mathrm{TE}_{0}$ sensing, show that the $\mathrm{TM}_{0}$ results are qualitatively very similar. The maximum sensitivity values with $p=2 / a_{c}$ are represented in Fig. 10. As compared with Fig. 8, the maximum sensitivity is also attained at $a_{c}=a_{s}$, but it peaks sharper as the guidance increases. Fig. 11 shows that the $\mathrm{TM}_{0}$ maximum sensitivity condition takes place further away from the cutoff than for the $\mathrm{TE}_{0}$ case.

In order to evaluate the effect of the measurand index on the maximum sensitivity conditions for the $\mathrm{TM}_{0}$ mode, we have calculated expressions (13), (17), and (18) for a number of different $\varepsilon_{f}$ and $\varepsilon_{c}$ values contained in a domain limited by $1.3<n_{c}<1.7$ and $1.3<n_{f}<1.7$. This domain of variation is wide enough to correspond to most possible cases in sensing involving a biological layer and a liquid cover. The two most extreme cases are reported here. The first corresponds to $n_{c}=1.7$ and $n_{f}=1.3$, thus to $p=2.71 / a_{c}$. The second case corresponds to $n_{c}=1.3$ and $n_{f}=1.7$, thus $p=1.585 / a_{c}$. The results show that the $X_{s}$ and the $n_{g} w / \lambda$ charts are very much the same as those of Fig. 11. The general shape of the maximum sensitivity surface reflects the same tendencies as those of Fig. 10 with $p=2 / a_{c}$. However, the value of the sensitivity changes significantly; Fig. 12 shows the maximum sensitivity surface with $p=2.71 / a_{c}$ corresponding to $\varepsilon_{f}<\varepsilon_{c}$. Although the maximum sensitivity takes place for neighboring values of $X_{s}$ and $n_{g} w / \lambda$, the sensitivity is

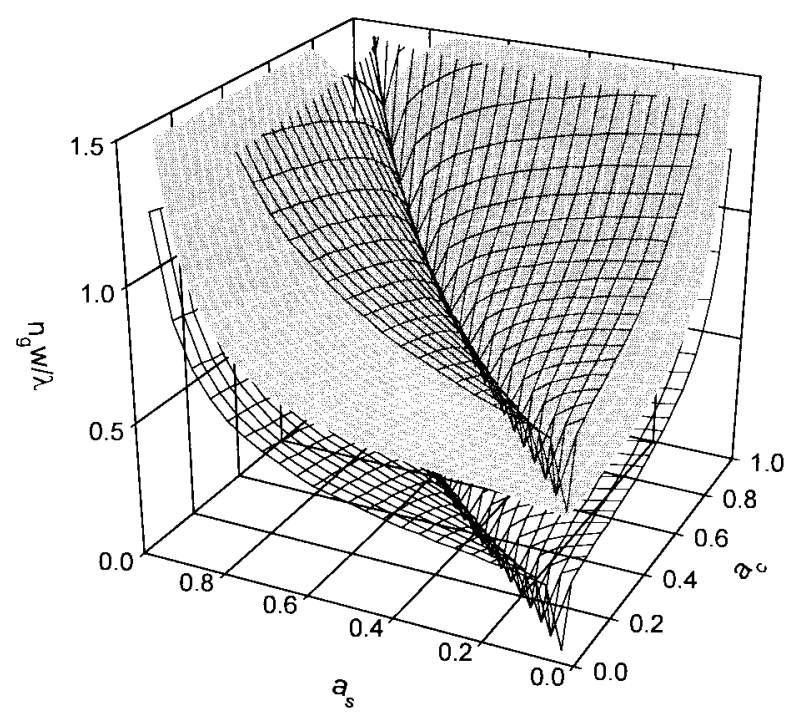

Fig. 11. Optical thickness $n_{g} w / \lambda$ ensuring the maximum sensitivity for $\mathrm{TM}_{0}$ surface sensing versus $a_{s}$ and $a_{c}$, corresponding to $p=2 / a_{c}$. The cutoff condition for the $\mathrm{TM}_{0}$ and the $\mathrm{TM}_{1}$ modes are indicated by the mesh.

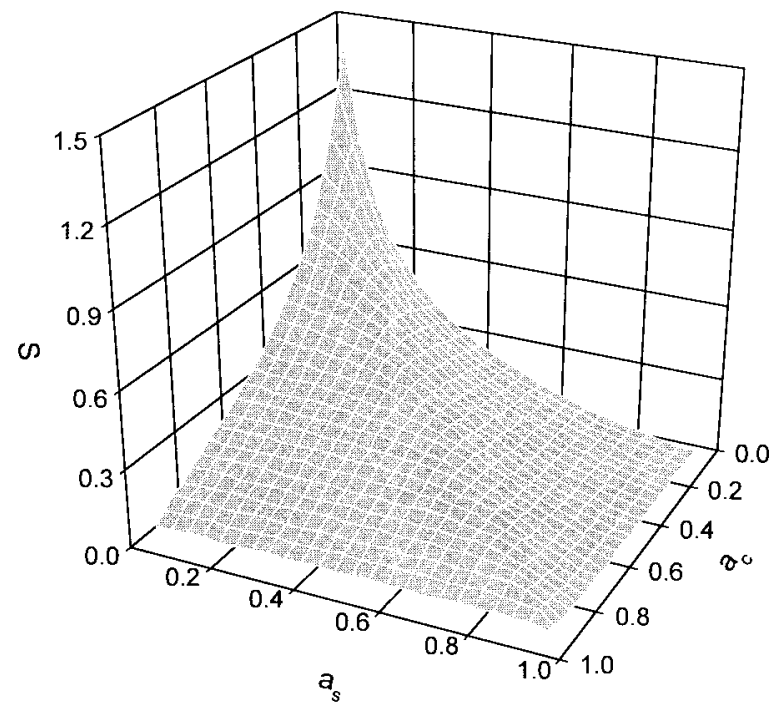

Fig. 12. Maximum achievable sensitivity for $\mathrm{TM}_{0}$ surface sensing versus $a_{s}$ and $a_{c}$, corresponding to $p=2.71 / a_{c}$, i.e., $\varepsilon_{f}<\varepsilon_{c}$.

roughly $50 \%$ higher than in the $p=2 / a_{c}$ case. Fig. 13 shows $S_{\max }$ for $p=1.585 / a_{c}$ corresponding to $\varepsilon_{f}>\varepsilon_{c}$; where the maximum sensitivity is roughly $80 \%$ of that for $p=2 / a_{c}$ although the optogeometrical conditions for it are very close.

Because of the nonnegligible dependence of the maximum achievable TM-sensitivity on the thin-film index it is interesting to make a parametrized comparison between $\mathrm{TE}_{0}$ and $\mathrm{TM}_{0}$ mode sensitivities in order to make the right polarization choice in every sensing configuration. The results of the comparison are reported in the chart of Fig. 14. The abscissa and ordinate of the chart represent $a_{c}$ and $a_{s}$, respectively. Every basic sensor structure with its defined substrate, guide and cover index represents a point in the chart. The $\left(a_{s}, a_{c}\right)$ plane is divided into two zones by a solid line for each value of the parameter $\varepsilon_{c} / \varepsilon_{f}$. The zone at the left of the line corresponds to the sensing structures where the optimal $S_{\mathrm{TM}}$ 


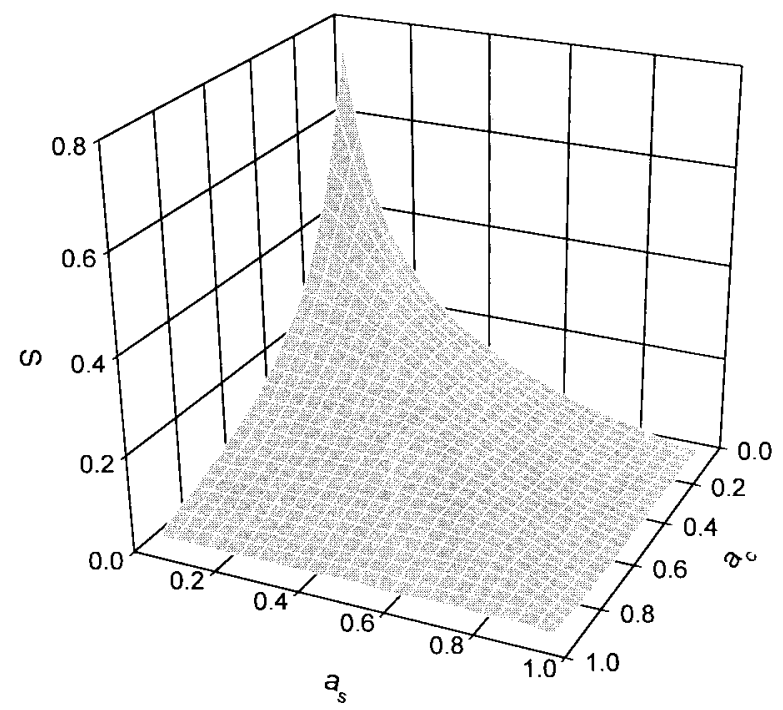

Fig. 13. Maximum achievable sensitivity for $\mathrm{TM}_{0}$ surface sensing versus $a_{s}$ and $a_{c}$, corresponding to $p=1.585 / a_{c}$, i.e., $\varepsilon_{f}>\varepsilon_{c}$.

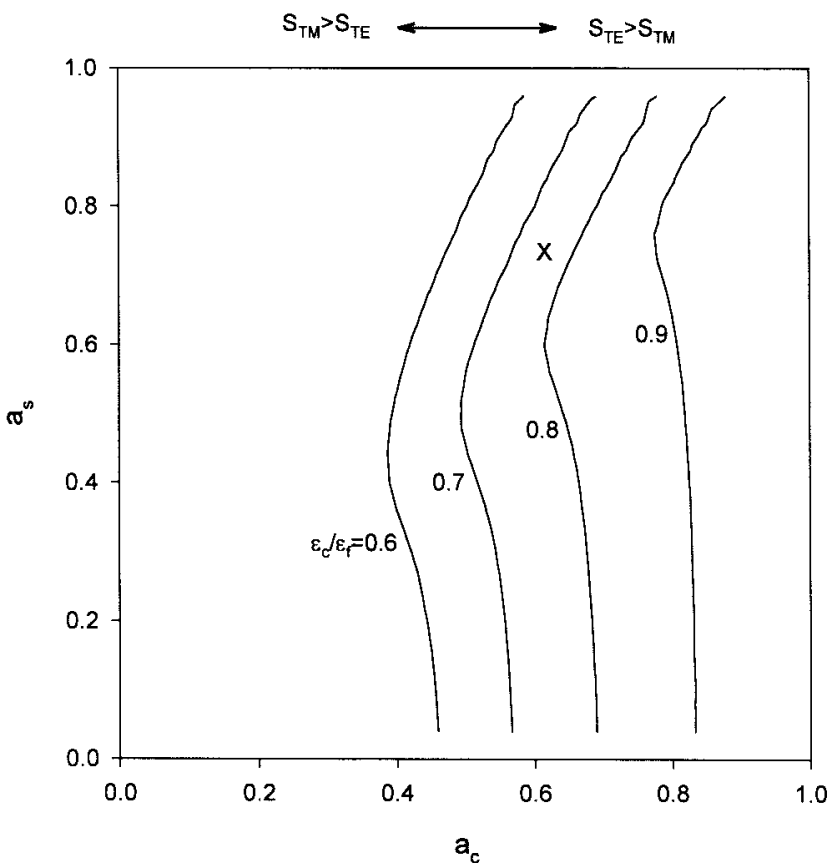

Fig. 14. $\left(a_{c}, a_{s}\right)$ plane with parametric lines $\varepsilon_{c} / \varepsilon_{f}$ dividing the plane in a left zone where $S_{\mathrm{TM}}>S_{\mathrm{TE}}$ and a right zone where $S_{\mathrm{TE}}>S_{\mathrm{TM}}$ in the surface sensing scheme.

is larger than the optimal $S_{\mathrm{TE}}$; the zone at the right of the line is for structures where $S_{\mathrm{TE}}>S_{\mathrm{TM}}$. It is interesting to note that $S_{\mathrm{TM}}$ is always larger than $S_{\mathrm{TE}}$ if $\varepsilon_{c} / \varepsilon_{f} \geq 1$, i.e., in case the measurand film index is lower than that of the solvent.

The use of the chart of Fig. 14 is illustrated by the concrete example of a silica substrate $\left(n_{s}=1.45\right)$, a $\mathrm{SiON}$ or $\mathrm{SiO}_{2}-$ $\mathrm{TiO}_{2}$ sol-gel guide $\left(n_{g}=1.7\right)$, an ultrathin film of index $n_{f}=1.6$, and a water cover, $n_{c}=1.33$. This structure is located by a cross in the $\left(a_{s}, a_{c}\right)$ plane: $a_{s}=0.73, a_{c}=$ 0.61 . For this structure the parametric line corresponding to $\varepsilon_{c} / \varepsilon_{f}=0.69$ is located between the two lines for $\varepsilon_{c} / \varepsilon_{f}=0.6$ and $\varepsilon_{c} / \varepsilon_{f}=0.7$, which are indicated in the figure. The cross is clearly at the right side of this line which means that the maximum sensitivity of the $\mathrm{TE}_{0}$ mode is larger than the maximum sensitivity of the $\mathrm{TM}_{0}$ mode.

As a conclusion, if the designer is free to use any sensing polarization, the structure should first be located in the $\left(a_{c}, a_{s}\right)$ plane of Fig. 14, and with respect to the parametric line $\varepsilon_{c} / \varepsilon_{f}$ corresponding to situation being considered. Then, if the TE polarization is the more sensitive one, the charts of Figs. 7-9 can be straightforwardly used. Should the TM polarization be the more sensitive one, then expressions (12), (17), and (18) must be calculated exactly and the charts drawn corresponding to Figs. 10 and 11.

3) Toward the Grating-Coupled Sensor: The results obtained here in the surface sensing case could have also been obtained by requiring the square of the electric field amplitude at the guide-cover interface to be maximum [7]. This condition is also a condition for the maximum of the efficiency of a waveguide coupling grating at the same interface. Does this mean that the present results provide the optimum design condition for a complete grating coupled surface sensor? The answer is "no." The radiation efficiency of a waveguide grating coupler is not only determined by the electric field strength at the interface where the grating is located, but also depends on the self-interference of a radiated order upon its successive partial reflections at the waveguide walls. This effect can be very pronounced in the case of high guidance waveguides [10], which is precisely where the sensing sensitivity is the largest. This subject will be treated in another paper.

\section{CONCLUSION}

The presented normalized analysis of a slab waveguide evanescent-wave sensor gives analytical results which solve explicitly the problem of the optimization of all possible slabwaveguide sensors once and for all. The general results are represented in the form of a single universal chart for the TE and TM modes in both sensing schemes of homogeneous and surface sensing. This analysis has also revealed features of which the generality does have practical implications.

Concerning homogeneous sensing, we have the following:

- the achievement of large maximum sensitivity does not require large-guidance integrated optic technology;

- for TE modes the configurations exhibiting large maximum sensitivity correspond to the proximity of the cutoff;

- for TM modes the configuration of large sensitivity corresponds to a larger distance from the cutoff;

- in most practical cases, e.g., where $a_{c}<a_{s}$, it is advantageous to use the $\mathrm{TM}_{0}$ mode.

We have also pointed out that the same generalized formalism and results are valid in the more typical case of lossy structures and of absorptive sensors.

Concerning surface sensing, we have the following.

- The maximum sensitivity on the dielectric load of an ultrathin film for both polarizations increases with a decrease of the asymmetry parameters $a_{c}$ and $a_{s}$ (i.e., $\left.n_{g} \gg n_{c}, n_{s}\right)$, and is always maximum in a symmetric waveguide (i.e., $n_{c}=n_{s}$ ). This is a noticeable feature as compared to homogeneous sensing where a high maxi- 
mum sensitivity can be obtained regardless of the strength of the guidance.

- A definite maximum sensitivity exists, whatever the relationship between the cover index and the substrate index. This is again in contrast with the homogeneous sensing case where a definite maximum only exists when index sensing is performed in the cladding medium of the lower index.

- The TM mode sensing sensitivity maximum is always larger than that of the TE when $\varepsilon_{f}<\varepsilon_{c}$. In cases where the film index is larger than the cover index, the TE mode sensitivity can be larger than that of the TM, particularly in low guidance waveguides and when $n_{f}$ and $n_{c}$ differ substantially.

- Contrary to the case of homogeneous evanescent wave sensing, the optimum sensing case corresponds to a wellconfined mode with high field strength at the guide-cover interface. This ensures good guidance conditions and allows for a high coupling efficiency of a waveguide grating placed at the guide-cover interface.

The normalized analytical solutions obtained in the surface sensing case can be applied to all slab-waveguide sensors where the measurand variation translates into a change of thickness or refractive index of an ultrathin layer at the waveguide surface. Similarly, the present results can readily be extended to the case of an absorbtive ultrathin transduction layer.

\section{ACKNOWLEDGMENT}

The authors are very grateful to P. V. Lambeck for his meaningful comments and useful suggestions on the contents of the paper and for reading the manuscript.

\section{REFERENCES}

[1] R. Cush, J. M. Cronin, W. J. Stewart, C. H. Maule, J. Molloy, and N. J. Goddard, "The resonant mirror: A novel optical biosensor for direct sensing of bio-molecular interactions, Part I: Principle of operation and associated instrumention," Biosensors Bioelectron., vol. 8, pp. 347-353, 1993.

[2] A. Bernard and H. R. Bosshard, "Real-time monitoring of antigenantibody recognition on a metal oxide surface by an optical grating coupler sensor," European J. Biochem., vol. 230, pp. 416-423, 1995.

[3] K. Tiefenthaler and W. Lukosz, "Sensitivity of grating couplers as integrated-optical chemical sensors," J. Opt. Soc. Amer. B., vol. 6, pp. 209-220, 1989.
[4] H. J. M. Kreuwel, "Planar waveguide sensors for the chemical domain," Ph.D. dissertation, Univ. Twente, The Netherlands, 1988.

[5] O. Parriaux, in Fiber Optic Chemical Sensors and Biosensors, O. Wolfbeis, Ed. Boca Raton, FL: CRC Press, 1991, vol. 1, pp. 111-192.

[6] D. Freiner, R. E. Kunz, D. Citterio, U. E. Spichiger, and M. T Gale, "Integrated optic sensors based on refractometry of ion-selective membranes," in Proc. EUROPTRODE II, Florence, Italy, Apr. 19-21, 1994; also see, Sensors \& Actuators B, vol. 29, pp. 277-285, 1995.

[7] O. Parriaux and P. Dierauer, "Normalized expressions for the optical sensitivity evanescent wave sensors," Opt. Lett., vol. 19, pp. 508-510, 1994.

[8] N. F. Hartman, D. Campbell, and M. Gross, "Waveguide interferometer configurations," in Proc. IEEE-LEOS'88, Santa Clara, CA, 1988, pp. 298-299.

[9] Ch. Fattinger, "Integrated optic biochemical sensors," in Proc. ECIO'95, Delft, The Netherlands, Apr. 1995, pp. 439-444.

[10] O. Parriaux, V. A. Sychugov, and A. V. Tishchenko, "Coupling gratings as waveguide functional elements," Pure Appl. Opt., vol. 5, pp. 453-469, 1996.

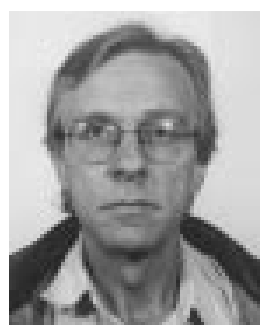

Olivier Parriaux (A'95) studied physics and received the $\mathrm{Ph} . \mathrm{D}$. degree in microwaves from the Federal Institute of Technology, Lausanne, Switzerland.

For three years, he was a Postdoctoral member at the University College London, U.K., and at the Moscow Lebedev Institute, Russia, in the field of fiber and integrated optics. From 1980 to 1983, he was Maitre de Conference at the Polytechnical Institute of Grenoble, France. In 1983, he joined CSEM Swiss Center for Electronics and Microtechnology, where he led the development of early industrial applications of fiber and integrated optics for sensors and microsystems. Through his active European commitment, he was Chairman of the 6th ECIO Conference and has been the manager of large European projects- the last one was EU-922 FOTA which he has been leading recently from Friedrich-Schiller-University Jena, Germany, where he is an Invited Professor. He is now Directeur de Recherche CNRS at Saint-Etienne University, France and keeps a docentship at Twente University, Enschede, The Netherlands. His current interests are in the search of new functionalities of waveguide and waveguideless gratings for their exploitation in sensors and microsystems.

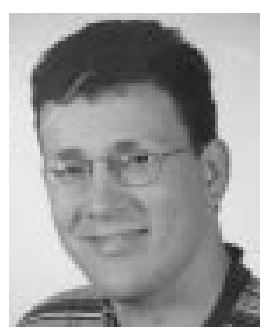

G. J. Veldhuis was born in Nieuwolda, The Netherlands, in 1970. He received the M.Sc. degree in applied physics from the University of Twente, The Netherlands, in 1994. He is presently working toward the Ph.D. degree in the field of integrated optics for sensor applications. 